\title{
INCONTINÊNCIA URINÁRIA EM MULHERES: BREVE REVISÃO DE FISIOPATOLOGIA, AVALIAÇÃO E TRATAMENTO
}

\section{URINARY INCONTINENCE IN WOMEN: A BRIEF REVIEW OF THIS PATHOPHYSIOLOGY, EVALUATION AND TREATMENT}

\author{
Fernando José Leopoldino Fernandes CÂNDIDO'1, Thiago MATNEI', Letícia \\ Carollyne GALVÃO', Vamilly Leal de Jesus SANTOS', Melina Chaves dos SANTOS', \\ Andrey Biff SARRIS', Bernardo Passos SOBREIRO²
}

\section{1 - Acadêmicos de Medicina da Universidade Estadual de Ponta Grossa (UEPG), Ponta Grossa, PR, Brasil. \\ 2 - Médico Urologista, Doutor em Clínica Cirúrgica e Professor Adjunto de Urologia da Universidade Estadual de Ponta Grossa (UEPG), Ponta Grossa, PR, Brasil. \\ Autor para correspondência: Email: fjcandido2006@hotmail.com}

\section{RESUMO:}

A incontinência urinária (IU) é definida como qualquer perda involuntária de urina que gere desconforto social ou higiênico para a paciente e possa ser objetivamente demonstrado. Estudos indicam que até $40 \%$ das mulheres americanas tem algum grau de IU. Para que os pacientes mantenham uma capacidade adequada de continência urinária, é necessário que diversos músculos, nervos simpáticos, parassimpáticos e somáticos atuem conjunta e harmonicamente, sendo que a falha de qualquer uma dessas estruturas pode culminar na perda involuntária de urina. Considerando-se os distintos mecanismos fisiopatológicos e as diferentes clínicas possíveis da IU, ela pode ser subdividida em cinco tipos básicos: a incontinência de esforço, de urgência, mista, paradoxal ou contínua. Esse distúrbio é mais comumente encontrado em mulheres caucasianas e com história familiar positiva, o que demonstra a participação de fatores hereditários em sua etiologia. Dentre os fatores de risco ambientais, podemos citar: idade avançada, prática de atividades físicas de alto impacto, grande paridade, obesidade, tabagismo e presença de algumas doenças crônicas, como diabetes mellitus. De forma geral, os episódios de incontinência são responsáveis por grande constrangimento social das pacientes acometidas, podendo comprometer suas vidas na esfera social, psicológica, física e econômica. Ainda assim, muitas pacientes não compreendem a IU como uma doença e acreditam que se trate apenas de uma complicação natural do ato de envelhecer. Isso é preocupante, pois mascara a real gravidade do problema e faz com que a IU seja subestimada e negligenciada por muitos profissionais de saúde. Nas últimas décadas, diversos tratamentos conservadores foram propostos, como terapias farmacológicas, utilização de exercícios específicos, biofeedback e estimulação elétrica ou magnética. Apesar disso, a cirurgia ainda é considerada o principal método terapêutico para muitas das pacientes acometidas pela IU.

Palavras-chave: Incontinência Urinária; Saúde da Mulher; Revisão.

\section{ABSTRACT:}

Urinary incontinence (UI) is defined as the involuntary loss of urine that causes social or hygienic issues and can be objectively demonstrated. Some papers indicate that about $40 \%$ of American women present some degree of UI. In order for urinary continence be 
maintained, several muscles, sympathetic, parasympathetic and sensorial nerves must be harmonically working. If any of these structures fail in its functions, UI may be developed. Considering the several distinct pathophysiological mechanisms, the UI can be subdivided in five basic types: stress, urge, mixed, paradoxical and continuous incontinence. This disorder is more frequently found in Caucasian women with a positive familiar history, which demonstrates that hereditary factors play a role in its etiology. Others possible risk factors may include: advanced age, high-impact physical activities, parity, obesity, smoking and some chronic diseases, such as diabetes. The UI episodes are generally responsible for great social embarrassment of the affected patients, and may compromise their social, psychological, physical and economic lives. Still, many patients do not comprehend UI as a disease and understand it as a natural complication of the aging process. This is worrisome, since it masks the real severity of the UI problem and may lead the health professionals to underestimate or neglect the problem. In the last few decades, several conservative treatment options have been proposed, such as pharmacological therapies, kinesiotherapy, biofeedback and electrical/magnetic stimulation. However, the surgical approach is still considered the main therapeutic method for many of the UI patients

Key-words: Urinary Incontinence; Women's Health; Review.

\section{INTRODUÇÃO}

A incontinência urinária (IU) pode ser definida como qualquer perda involuntária de urina que determine desconforto social ou higiênico e seja demonstrável de modo objetivo (ABRAMS et al., 2002). Estudos indicam que até 40\% das mulheres americanas tem algum grau de IU (ANGER et al., 2006). Uma parcela delas ainda apresenta esse problema com interferência em atividades cotidianas, como nas relações interpessoal e sexual. Apesar disso, muitas ainda não procuram auxílio médico. (KELLEHER et al., 1995; SUSKIND et al., 2016).

Mulheres geralmente apresentam IU de estresse ou urgi-incontinência, enquanto homens tendem a apresentar problemas prostáticos e, portanto, problemas relacionados à incontinência contínua. É válido lembrar também que diversas medicações, especialmente relacionadas a próstata, podem provocar um quadro de IU. (KHANDELWAL et al., 2013).

\section{METODOLOGIA}

Foi realizada consulta à literatura acadêmica através das bases de dados eletrônicos "PUBMED" e "SCIELO" durante os meses de julho e agosto de 2017. Nas bases, foram utilizados os descritores "incontinência urinária" na língua portuguesa e inglesa, assim como seus qualificadores - prevenção e controle, diagnóstico, tratamento farmacológico, terapia, entre outros. Foram lidos os resumos dos principais artigos considerados adequados, atuais e importantes para a temática. Após a leitura dos resumos, 
os artigos com referenciação mais embasada foram considerados apropriados e incluídos nessa revisão.

\section{FISIOPATOLOGIA DA INCONTINÊNCIA URINÁRIA}

O armazenamento de urina e posterior esvaziamento da bexiga é um processo fisiologicamente complexo e, para que possa ocorrer de forma adequada, é necessário que diferentes músculos, nervos parassimpáticos, simpáticos, somáticos e sensoriais trabalhem conjuntamente. A falha de qualquer uma dessas estruturas pode culminar no desenvolvimento de IU (BARACHO, 2002; GIRÃO, 1997). Considerando-se isso, a IU pode ser dividida em cinco tipos clínicos distintos, de acordo com o mecanismo fisiopatológico envolvidos (TABELA 1).

Tabela 1. Tipos de Incontinência Urinária, Clínica e Mecanismos

\begin{tabular}{|c|c|c|}
\hline Tipo & Clínica & Mecanismo \\
\hline $\begin{array}{l}\text { Incontinência urinária de } \\
\text { esforço }\end{array}$ & $\begin{array}{l}\quad \text { Perda involuntária de urina } \\
\text { ocasionada por situações que levem } \\
\text { ao aumento da pressão intravesical, } \\
\text { como tosses, espirros, levantamento } \\
\text { de peso, caminhadas, dentre outros. }\end{array}$ & $\begin{array}{l}\text { Causada pela redução da pressão } \\
\text { uretral, que se torna incapaz de } \\
\text { impedir a saída de urina durante a } \\
\text { realização de atividades que } \\
\text { aumentem a pressão intravesical, } \\
\text { como tosses e espirros. }\end{array}$ \\
\hline $\begin{array}{c}\text { Incontinência urinária de } \\
\text { urgência }\end{array}$ & $\begin{array}{l}\text { Necessidade súbita, intensa e } \\
\text { imperiosa de urinar. O paciente } \\
\text { perde urina involuntariamente, pois } \\
\text { não tem tempo suficiente para } \\
\text { chegar ao banheiro. }\end{array}$ & $\begin{array}{l}\text { Decorre de distúrbios neurológicos } \\
\text { sensitivos ou hiperatividade motora do } \\
\text { músculo detrusor. }\end{array}$ \\
\hline $\begin{array}{l}\text { Incontinência urinária } \\
\text { mista }\end{array}$ & $\begin{array}{c}\text { Características simultâneas de } \\
\text { incontinência urinária de esforço e } \\
\text { de urgência. }\end{array}$ & $\begin{array}{c}\text { Mecanismos fisiopatológicos mistos da } \\
\text { incontinência urinária de esforço e de } \\
\text { urgência. }\end{array}$ \\
\hline $\begin{array}{l}\text { Incontinência urinária } \\
\text { paradoxal }\end{array}$ & $\begin{array}{c}\text { Paciente possui vontade de urinar, } \\
\text { mas apenas consegue eliminar } \\
\text { gotas de urina. }\end{array}$ & $\begin{array}{l}\text { A bexiga não é capaz de ser } \\
\text { esvaziada, por problemas neurológicos } \\
\text { ou obstrutivos infravesicais. Quando } \\
\text { ela está completamente cheia, a urina } \\
\text { transborda involuntariamente. }\end{array}$ \\
\hline $\begin{array}{l}\text { Incontinência urinária } \\
\text { contínua }\end{array}$ & Perda urinária constante. & $\begin{array}{l}\text { Causada por graves lesões ao sistema } \\
\text { esfincteriano, podendo ser secundária } \\
\text { a ressecções pélvicas ou traumas } \\
\text { genitais. A pressão uretral torna-se } \\
\text { incapaz de impedir o fluxo urinário. }\end{array}$ \\
\hline
\end{tabular}

FONTE: BARACHO, 2002; GIRÂO, 1997; KOBASHI, 2012) 
Didaticamente, as causas mais comumente encontradas de IU podem ser agrupadas em dois grupos: (1) as resultantes de alterações vesicais primárias ou secundárias; e (2) as decorrentes de distúrbios uretrais. As alterações vesicais são principalmente representadas pela hiperatividade da musculatura detrusora, caracterizada por contrações involuntárias desse músculo, que culminam na expulsão involuntária da urina armazenada. A hiperatividade do detrusor pode ser subdividida em dois tipos: fásica e terminal. Na hiperatividade fásica, a amplitude das contrações crescem de acordo com o volume vesical e podem, ou não, levar à IU. Por outro lado, na hiperatividade terminal, há uma única e involuntária contração do detrusor, que não pode ser suprimida e invariavelmente resulta em IU, com esvaziamento completo da bexiga. (BARACHO, 2002; GIRÃO, 1997; KOBASHI, 2012; WOOD et al., 2014).

Outro exemplo comum de alteração vesical é a redução da complacência vesical. Em situações fisiológicas, a bexiga apresenta alta complacência. Ou seja, ela é capaz de acumular grandes quantidades de urina, sem que sua pressão interna seja consideravelmente elevada. Todavia, quando essa propriedade é reduzida, mesmo pequenas alterações de volume são capazes de gerar grandes pressões intravesicais, estimulando o desejo miccional do paciente. Ainda que essa situação possa decorrer de alterações elásticas da bexiga, ela é mais comumente relacionada a lesões neurológicas motoras baixas, como espinha bífida e síndrome da cauda equina. Apesar da relevância desse mecanismo fisiopatológico, cabe ressaltar que ainda não existem métodos internacionais e padronizados para classificar a complacência vesical em alta, média ou baixa. (BARACHO, 2002; GIRÃO, 1997; KOBASHI, 2012; WOOD et al., 2014).

O principal exemplo de incontinência resultante de distúrbios uretrais é a IU de esforço, que resulta de uma insuficiência esfincteriana. Nessa situação, a pressão de resistência da musculatura uretral torna-se reduzida e, com isso, o fechamento da uretra fica incapaz de impedir o fluxo urinário, ainda que a pressão intravesical esteja normal. Diversos fatores podem explicar essa insuficiência esfincteriana, como alterações anatômicas e defeitos no esfíncter intrínseco da uretra. A redução nos níveis séricos de estrogênio, por exemplo, reduz a vascularização periuretral e promove a atrofia da musculatura local, estimulando o desenvolvimento desse tipo de incontinência. Esse é um dos principais motivos pelos quais a IU é mais comumente encontrada em mulheres idosas. Apesar disso, diversos outros fatores também já foram correlacionados com distúrbios 
uretrais, como fraqueza anatômica, idade avançada, cirurgias pélvicas prévias e obesidade. (BARACHO, 2002; KOBASHI, 2012;3 WOOD et al., 2014).

Por fim, ainda que sejam clinicamente menos comuns, outros mecanismos fisiopatológicos também podem levar ao desenvolvimento da IU. No caso da incontinência paradoxal, por exemplo, os pacientes apresentam um transbordamento de urina quando a bexiga apresenta-se mais cheia do que sua capacidade máxima. Esse processo pode resultar de processos obstrutivos do trato urinário inferior.

\section{FATORES DE RISCO PARA INCONTINÊNCIA URINÁRIA}

Ainda que a IU seja mais frequentemente encontrada na população feminina, sua prevalência exata e seus fatores de risco são difíceis de ser determinados, pois as metodologias empregadas variam significativamente entre os trabalhos realizados. Nessa revisão, apenas serão abordados os fatores de risco mais comumente comentados na literatura médica. Sabe-se que essa doença possui uma prevalência maior em mulheres caucasianas e que está 2,6 vezes mais presentes em mulheres com um histórico familiar de incontinência (HIGA et al., 2008; WOOD et al., 2014). Dessa forma, ainda que os fatores de risco ambientais sejam relevantes para o desenvolvimento desse distúrbio, os fatores genéticos também merecem ser levados em consideração.

A idade é aceita como um dos principais fatores de risco, pois a prevalência da IU aumenta consideravelmente após a menopausa (HIGA et al., 2008; KOBASHI, 2012; WOOD et al., 2014). Acredita-se que isso se deva principalmente à redução do estrogênio sérico em mulheres nessa faixa etária. Como previamente mencionado, os menores níveis desse hormônio culminam em atrofia da musculatura uretral e facilitam o desenvolvimento da IU. Outros fatores também podem estar correlacionados à maior prevalência da incontinência em populações idosas, como a o aumento no índice de massa corporal nessa faixa etária e a maior presença de doenças crônicas, como diabetes e doença pulmonar obstrutiva crônica. (HIGA et al., 2008).

Ainda que haja divergências sobre o assunto, alguns autores têm colocado que a prática de atividades físicas de alto impacto também está correlacionada com o desenvolvimento de IU. Em teoria, esses exercícios causariam um aumento da pressão intra-abdominal, o que, por sua vez, sobrecarregaria os órgãos pélvicos e danificaria as 
estruturas musculares adjacentes, responsáveis por oferecer suporte a esses órgãos (CAETANO et al., 2007). De fato, Nygaard et al (1994) em um estudo conduzido com 156 atletas nulíparas demonstrou que $28 \%$ delas apresentavam perda involuntária de urina durante a realização de atividades físicas. Os esportes de alto impacto, como ginástica, basquete e tênis foram identificados como os esportes com maior número de incontinentes, enquanto esportes considerados de baixo impacto, como natação, foram responsáveis por apenas $4,5 \%$ das queixas de incontinência.

A obesidade tem sido descrita como um fator causador e agravante da IU simultaneamente. Ao exemplo do que ocorre com os esportes de alto impacto, a obesidade também aumenta a pressão intra-abdominal e intravesical, alongando e enfraquecendo a musculatura do assoalho pélvico (CASTRO et al., 2012; KOBASHI, 2012; WOOD et al., 2014). Nesse contexto, Castro et al (2012) comprovaram que a redução de peso é benéfico para as mulheres obesas e incontinentes. Em seu trabalho, demonstrou-se que $70,59 \%$ das mulheres obesas deixavam de apresentar IU quando submetidas à cirurgia bariátrica. Nessa pesquisa, a força muscular do assoalho pélvico, mensurada através da escala Oxford modificada, aumentou significativamente em relação ao pré-operatório. Em seu estudo, é interessante notar que mesmo perdas de peso pequenas, como de $5 \%$ a $10 \%$ do peso corporal, já foram capazes de resultar em melhorias na continência vesical das pacientes avaliadas. (CASTRO et al., 2012).

A paridade também tem sido mencionada como fator de risco para a IU em alguns estudos. Apesar disso, ainda há divergências literárias sobre o assunto. Alguns trabalhos demonstraram que o aumento do número de partos está diretamente correlacionado com a ocorrência de IU, enquanto outros afirmam que não há relação estatística suficientemente significativa para que essa relação se justifique. Além disso, ainda há autores que afirmam que o peso do recém-nascido também deve ser considerado como um fator de risco para o desenvolvimento dessa patologia, colocando que fetos mais pesados aumentariam ainda mais a pressão intra-abdominal materna (HIGA et al., 2008; KOBASHI, 2012).

Dentre as doenças crônicas correlacionadas com a IU, a diabetes mellitus é a mais comumente citada. Acredita-se que os elevados níveis glicêmicos sejam capazes de causar lesões nos nervos autônomos da bexiga, prejudicando o mecanismo de enchimento e esvaziamento vesical. Além disso, a glicosúria poderia causar um aumento na frequência urinária, agravando ainda mais o quadro de IU nesse grupo de pacientes (HIGA et al., 2008; 
WANG et al., 2015).

Por fim, também se discute a possibilidade do tabagismo ser um fator de risco. $\mathrm{O}$ fumante frequentemente apresenta uma tosse mais violenta, podendo afetar direta ou indiretamente a bexiga e a uretra, danificando o mecanismo esfincteriano e propiciando à IU. O monóxido de carbono e a nicotina presentes no tabaco também agem reduzindo os níveis de estrogênio disponíveis, fator já relacionado previamente com a IU. (HIGA et al., 2008; SANGSAWANG, 2014).

\section{AVALIAÇÃO DA INCONTINÊNCIA URINÁRIA}

O impacto na qualidade de vida de um paciente incontinente pode ser avaliado através de questionários, como o King's Health Questionnaire (KHQ). Essas avaliações visam identificar a presença de sintomas de IU, bem como seus impactos relativos na vida do doente. O questionário citado já possui uma versão traduzida e validada para o idioma português.

Há um consenso na literatura internacional de que os episódios de IU são responsáveis por grande constrangimento social, podendo levar os incontinentes a desenvolverem quadros de depressão, isolamento social, estresse, baixa autoestima e sentimento de vergonha. Por esse motivo, coloca-se que essa desordem afeta seus portadores na esfera social, psicológica, física e econômica. (OLIVEIRA et al., 2009; PITANGUI et al., 2012).

Nota-se que, ainda que a doença seja desagradável para as pacientes, muitas mulheres idosas com essa situação não buscam auxílio profissional, pois a consideram uma consequência natural do envelhecimento. Oliveira et al (2009) por exemplo, comprovaram que grande parte das mulheres com incontinência urinária, quando questionadas, classificam a própria saúde como sendo "boa" ou "normal", o que evidencia que a população, de forma geral, não enxerga a IU como uma doença. Esse fato é preocupante, pois pode mascarar a real gravidade do problema, visto que faz com que a IU seja subestimada - e até mesmo negligenciada - por diversos profissionais de saúde. (OLIVEIRA et al., 2009).

Ainda que os episódios de incontinência sejam desagradáveis, várias formas de abordagem terapêutica estão atualmente disponíveis para essas pacientes e apresentam 
bons resultados clínicos. Essas técnicas serão brevemente resumidas adiante. Considerando-se que muitas pacientes podem ter a perda involuntária de urina reduzida ou evitada, consideramos que a criação de políticas educativas que possam informar a população de que incontinência urinária não é uma condição normal poderia ser benéfica para diversos indivíduos. Além disso, estimulamos os médicos a questionarem ativamente as pacientes sobre possíveis episódios de incontinência urinária, quando houver claros fatores de risco associados.

\section{TRATAMENTO DA INCONTINÊNCIA URINÁRIA}

\subsection{Tratamento Conservador}

Diversas formas de tratamento conservador foram sugeridas para tratar IU, incluindo medicamentos, exercícios específicos, biofeedback, técnicas de estimulação elétrica ou magnética, dentre muitas outras. Muitos desses métodos, ainda que antigos, permanecem controversos na literatura médica, visto que ainda existem dúvidas quanto aos reais benefícios e efeitos colaterais de suas utilizações. Apesar disso, cabe ressaltar que, independente do método terapêutico recomendado, é importante que a paciente incontinente seja submetida a uma reeducação comportamental. Ela deve estabelecer um ritmo miccional frequente - de hora em hora, por exemplo - e buscar aumentar o intervalo de tempo entre as micções. (OLIVEIRA et al., 2007; ROBINSON et al., 2014).

Dentre os medicamentos, a terapia de reposição hormonal com estrógenos tem sido descrita como uma terapêutica eficaz no controle da IU em mulheres na pósmenopausa. A reposição desse hormônio agiria sobre a uretra, aumentando sua pressão de fechamento e facilitando a reposta a agonistas dos receptores $\alpha$-adrenérgicos. Ainda que diversos estudos tenham demonstrado melhora clínica dos sintomas com esse tratamento, é possível que isso se deva ao fato de que o estrógeno aumenta a sensação de bem-estar geral do usuário. Os potenciais benefícios devem ser avaliados sob a luz dos possíveis riscos dessa terapia, visto que alguns trabalhos constataram que a utilização desse hormônio está relacionada com um maior risco de desenvolver neoplasias mamárias e uterinas. Por esse motivo, o emprego dessa terapêutica para o controle da IU ainda é considerado controverso e sua dosagem ideal, via de administração e duração do 
tratamento nunca foram nitidamente esclarecidos. (CASTRO et al., 2015; KOBASHI, 2012; LEGENDRE et al., 2013).

Os agonistas $\alpha$-adrenérgicos também foram sugeridos como potenciais fármacos para controlar a sintomatologia da IU. A princípio, eles agiriam estimulando a contração da musculatura uretral e aumentado a pressão de fechamento da uretra, o que impediria a perda involuntária de urina. Ainda que a teoria tenha sido bem estabelecida, os trabalhos demonstraram apenas uma cura subjetiva. (CASTRO et al., 2015; KOBASHI, 2012).

Ainda no contexto da utilização de neurotransmissores periféricos para reverter a IU, a possibilidade de utilizar antidepressivos tricíclicos como tratamento também foi proposta. Acredita-se que eles inibiriam a recaptação da noradrenalina nas terminações nervosas adrenérgicas da uretra, o que potencializaria seus efeitos contráteis sobre a musculatura local. Todavia, ainda não foram realizados estudos conclusivos sobre o assunto e a real eficácia desse tratamento não pode ser confirmada. (CASTRO et al., 2015; KOBASHI, 2012).

A cinesioterapia tem sido proposta como uma terapêutica não farmacológica para a IU. Ela consiste basicamente em exercícios físicos repetitivos que objetivam reforçar a resistência uretral e fortalecer os músculos responsáveis pela sustentação dos órgãos pélvicos. Essas atividades, popularmente conhecidas como exercícios de Kegel, consistem basicamente na realização de rápidas contrações da musculatura pélvica. As técnicas têm se mostrado eficazes, sendo que diversos autores reportaram que, quando utilizadas, elas podem levar a uma melhora de $70 \%$ dos episódios de perdas urinárias. A principal dificuldade dessa abordagem é o fato de que a maior parte dos pacientes submetidos a ela desconhecem os músculos sendo exercitados. Isso pode levá-los a contrair músculos errôneos, como reto abdominal ou glúteo máximo. Assim sendo, o tipo de exercício a ser indicado dependerá da força atual do assoalho pélvico, da capacidade de reconhecimento da musculatura por parte do paciente e do grau da IU. (OLIVEIRA et al., 2007).

A técnica de biofeedback consiste na utilização de um dispositivo de monitoramento de eventos fisiológicos que, de outra forma, passariam desapercebidos pelo paciente. No caso da IU, a contração muscular é monitorada através de eletromiografia, o que permite que o paciente distinga a ação do músculo elevador do ânus e do reto abdominal (OLIVEIRA et al., 2007). Ao desenvolver a percepção correta de quais músculos está contraindo, o paciente torna-se capaz de desenvolver o controle voluntário desses 
músculos, através de programas de fortalecimento da musculatura agonista e antagonista. Alguns estudos chegam a demonstrar uma melhora de $82 \%$ nos episódios de incontinência com a utilização dessa abordagem. (LOPES et al., 2012).

Por fim, as técnicas de estimulação podem ser feitas através de estímulos elétricos ou magnéticos. A eletroestimulação é adquirida através da implantação de pequenos dispositivos intravaginais ou transanais, capazes de desenvolver determinada corrente elétrica e inibir o músculo detrusor. Essa inibição atua reduzindo o número de micções e aumentando, portanto, a capacidade vesical. Por outro lado, a estimulação magnética perianal é uma técnica mais recente, que consiste em despolarizar os nervos do assoalho pélvico e estimular a musculatura da região. Supostamente, sua utilização possui eficácia semelhante a estimulação elétrica. Ainda que ela apresente bases físicas e neurofisiológicas sólidas, a técnica ainda é relativamente recente e, portanto, apresenta poucos estudos definitivos capazes de avalia-la e validá-la. (OLIVEIRA et al., 2007).

\subsection{Tratamento Cirúrgico}

O tratamento cirúrgico é atualmente a principal terapêutica para a IU de esforço, ainda que seja reservado para os casos refratários ao tratamento conservador. A escolha da técnica mais apropriada para determinado caso sempre é um desafio, pois deve levar em consideração inúmeros fatores como: correto diagnóstico, índices de recidiva, integridade do sistema esfincteriano uretral e experiência do cirurgião. (KOBASHI, 2012; WOOD et al., 2014).

A cirurgia com uso de slings na uretra é considerada como terapia de primeira escolha em pacientes com IU de esforço por lesão do esfíncter intrínseco da uretra. Essa cirurgia consiste na inserção de uma fita abaixo da uretra da paciente, visando aumentar a resistência uretral e a sustentação do colo da bexiga. Os slings podem ser autólogos, como os provenientes da aponeurose do músculo reto abdominal, ou sintéticos, como telas TVT (tension-free vaginal tape). (FORD et al., 2015; JOVANOVIĆ et al., 2014).

Pacientes com hipermobilidade de uretra podem ser tratados com colpofixação retropúbica, um procedimento cirúrgico que objetiva recolocar a uretra proximal e o colo vesical em posição retropúbica, alta e fixa. Para isso, a técnica de Burch é a mais comumente utilizada, mas pode apresentar complicações como instabilidade vesical, 
hipercorreção, fibrose periutral e predisposição ao prolapso genital. Por esse motivo, o uso de slings superou a colpofixação e, em 1990, passou a ser a técnica mais utilizada no tratamento da IU no mundo todo. A colpofixação costuma ser reservada para pacientes que serão submetidos a cirurgias abdominais concomitantes ou estejam em locais que disponham de slings sintéticos. (FORD et al., 2015; JOVANOVIĆ et al., 2014).

\section{CONCLUSÃo}

Os episódios de incontinência urinária podem ser constrangedores e acarretar graves consequências para a qualidade de vida das pacientes acometidas, como isolamento do convívio social, depressão e baixa autoestima. Apesar disso, a doença é muitas vezes considerada como uma etapa normal do processo de envelhecimento, o que faz com que as queixas dessa desordem sejam negligenciadas pela população em geral e subdiagnosticada pelos profissionais de saúde. Considerando-se a grande variedade de terapias atualmente disponíveis e os bons resultados clínicos alcançados, torna-se relevante a criação de políticas educativas que possam informar a população sobre essa situação e orientá-los a buscar profissionais de saúde, caso necessário. Além disso, os médicos devem ser estimulados a questionar ativamente sobre possíveis episódios de perda urinária involuntária em pacientes com fatores de risco para esse tipo de incontinência.

\section{REFERÊNCIAS}

ABRAMS, P.; CARDOZO, L.; FALL, M.; GRIFFITHS, D.; ROSIER, P.; ULMSTEN, U.; et al. The standardisation of terminology of lower urinary tract function: report from the Standardisation Sub-committee of the International Continence Society. Am J Obstet Gynecol. 2002 Jul;187(1):116-26.

ANGER, J.T.; SAIGAL, C.S.; LITWIN, M.S. The prevalence of urinary incontinence among community dwelling adult women: results from the National Health and Nutrition Examination Survey. Journal of Urology. 2006; 175:601-604.

BARACHO, E. Fisioterapia aplicada à obstetrícia: aspectos de ginecologia e 
neonatologia. 3 ed. Rio de Janeiro: Medsi, 2002.

CAETANO, A.S.; TAVARES, M.C.G.C.F.; LOPES, M.H.B.M. Incontinência urinária e a prática de atividades físicas. Rev Bras Med Esporte vol.13 no.4 Niterói jul/ago 2007.

CASTRO, L.A.; SOBOTTKA, W.; BARETTA, G.; FREITAS, A.C.T. Efeitos da cirurgia bariátrica na função do assoalho pélvico. ABCD, Arq. Bras. Cir. Dig. vol.25 no.4 São Paulo out./dez. 2012.

CASTRO, R.A.; ARRUDA, R.M.; BORTOLINI, M.A. Female urinary incontinence: effective treatment strategies. Climacteric. 2015 Apr;18(2):135-41. doi: 10.3109/13697137.2014.947257.

FORD, A.A.; ROGERSON, L.; CODY, J.D.; OGAH, J. Mid-urethral sling operations for stress urinary incontinence in women. Cochrane Database Syst Rev. 2015 Jul 1;(7):CD006375. doi: 10.1002/14651858.CD006375.pub3.

GIRÃO, M.J.B.C. Uroginecologia. São Paulo, Editora Artes Médicas, 1997. p.33.

HIGA, R.; LOPES, M.H.B.M.; REIS, M.J. Fatores de risco para incontinência urinária na mulher. Rev Esc Enferm USP 2008; 42(1):187-92.

JOVANOVIĆ, M.; DŽAMIĆ, Z.; AĆIMOVIĆ, M.; et al. Trans-Obturator-Tape (T.O.T.) "outside-in" approach in surgical treatment of female stress urinary incontinence. Acta Chir lugosl. 2014;61(1):69-72.

KOBASHI, K.C. Evaluation of patients with urinary incontinence and pelvic prolapse. In: KAVOUSSI, L.R.; PARTIN, A.W.; NOVICK, A.C., et al. Campbell-Walsh Urology. New York, 11ํㅡ ed., 2012, p.1896-1908.

KELLEHER, C.J.; CARDOZO, L.D.; TOOZS-HOBSON, P.M. Quality of life and urinary incontinence. Current opinion in obstetrics \& gynecology. 1995 Oct; 7(5):404-408.

KHANDELWAL, C.; KISTLER, C. Diagnosis of urinary incontinence. Am Fam Physician. 
2013 Apr 15;87(8):543-50.

LEGENDRE, G.; RINGA, V.; FAUCONNIER, A.; FRITEL, X. Menopause, hormone treatment and urinary incontinence at midlife. Maturitas. 2013 Jan;74(1):26-30. doi: 10.1016/j.maturitas.2012.10.005.

LOPES, D.B.M.; PRACA, N.S. Incontinência urinária autorreferida no pós-parto: características clínicas. Rev. Esc. Enferm. Usp, 2012, vol.46, n.3, pp. 559-564.

NYGAARD, I.; THOMPSSON, F.L.; SVENGALIS, S.L. Urinary incontinence in elite nulliparous athletes. Obstet Gynecol. 1994;84:183-7.

OLIVEIRA, K.A.C.; RODRIGUES, A.B.C.; PAULA, A.B. Técnicas fisioterapêuticas no tratamento e prevenção da incontinência urinária de esforço na mulher. Revista Eletrônica F@pciência, Apucarana-PR, v.1, n.1, 31-40, 2007.

OLIVEIRA, S.G.; BATTISTI, B.Z.; SECCO, V.L.; POLESE, J.C. Avaliação da qualidade de vida de portadores de incontinência urinária. Revista Brasileira de Ciências do Envelhecimento Humano, Passo Fundo, jan./abr. 2009, 6(1):34-41.

PITANGUI, A.C.R.; SILVA, R.G.; ARAUJO, R.C. Prevalência e impacto da incontinência urinária na qualidade de vida de idosas institucionalizadas. Rev. Bras. Geriatr. Gerontol. 2012, vol.15, n.4, pp. 619-626.

ROBINSON, D.; CARDOZOWAN, L. Urinary incontinence in the young woman: treatment plans and options available. Womens Health (Lond). 2014 Mar;10(2):201-17. doi: $10.2217 /$ whe.14.1.

SANGSAWANG, B. Risk factors for the development of stress urinary incontinence during pregnancy in primigravidae: a review of the literature. Eur J Obstet Gynecol Reprod Biol. 2014 Jul; 178:27-34. doi: 10.1016/j.ejogrb.2014.04.010.

SUSKIND, A.M.; DUNN, R.L.; MORGAN, D.M., et al. A Screening Tool for Clinically Relevant Urinary Incontinence. Neurourol Urodyn. 2015 Apr; 34(4): 332-335. doi: 
$10.1002 /$ nau.22564

WANG, R.; LEFEVRE, R.; HACKER, M.R.; GOLEN, T.H. Diabetes, Glycemic Control, and Urinary Incontinence in Women. Female Pelvic Med Reconstr Surg. 2015 SepOct;21(5):293-7. doi: 10.1097/SPV.0000000000000193.

WOOD, L.N.; ANGER, J.T. Urinary incontinence in women. BMJ. 2014 Sep 15;349:g4531. doi: 10.1136/bmj.g4531. 